\title{
Forces due to structure in a thin liquid crystal film
}

\author{
R. G: Horn, J. N. Israelachvili and E. Perez (*) \\ Department of Applied Mathematics, Research School of Physical Sciences, The Australian National University, Canberra, \\ A.C.T. 2600, Australia
}

(Reçu le 20 juin 1980, accepté le 5 septembre 1980)

\begin{abstract}
Résumé. - On peut obtenir des renseignements sur la structure d'un film de cristal liquide qui sépare deux surfaces solides en mesurant les variations, en fonction de leur séparation, de la force qui s'exerce entre elles. Ici, ces mesures sont faites en utilisant des surfaces de mica moléculairement planes séparées par un cristal liquide, le $4^{\prime}$-n-pentyl 4-cyanobiphényle (5CB). L'orientation du 5CB est soit planaire soit homéotrope. La force est déterminée par la mesure de la flexion d'un ressort qui supporte une des feuilles de mica, et une technique optique est simultanément utilisée pour mesurer l'épaisseur du film avec une précision de $\pm(0,1-0,2) \mathrm{nm}$. Cette technique permet également de mesurer les indices de réfraction du film de cristal liquide et donc de déterminer la densité et le paramètre d'ordre moyens en fonction de son épaisseur.

On met en évidence trois types de forces, chacun reflétant un mode de structuration du cristal liquide près des surfaces de mica. Le premier provient d'une déformation élastique dans le cristal liquide; il est uniquement observé dans les films planaires twistés où les molécules de $5 \mathrm{CB}$ sont orientées dans des directions différentes sur les deux surfaces de mica. Le second, mesuré à la fois dans les structures planaires et homéotropes, est attribué à une augmentation du paramètre d'ordre près des surfaces. Ces deux forces sont répulsives et monotones, mesurables en dessous de $80 \mathrm{~nm}$. Enfin, il y a une force de courte portée (jusqu'à six couches moléculaires) qui oscille entre l'attraction et la répulsion en fonction de l'épaisseur. Ceci provient de la structuration des molécules en couches près de la surface solide. On observe ce phénomène dans les structures planaires, homéotropes, et également dans des liquides isotropes.
\end{abstract}

\begin{abstract}
Measurements of the force as a function of distance between two solids separated by a liquid crystal film give information on the structure of the film. We report such measurements for two molecularly smooth surfaces of mica separated by the nematic liquid crystal 4'-n-pentyl 4-cyanobiphenyl (5CB) in both the planar and homeotropic orientations at room temperature. The force is determined by measuring the deflection of a spring supporting one of the mica pieces, while an optical technique is used to measure the film thickness to an accuracy of $\pm(0.1-0.2) \mathrm{nm}$. The technique also allows the refractive indices of the nematic to be measured, and hence a determination of the average density and order parameter of the liquid crystal film as a function of its thickness. Three distinct forces were measured, each reflecting a type of ordering of the liquid crystal near the mica surfaces. The first one results from elastic deformation in the liquid crystal; it was only observed in a twisted planar sample where the 5CB molecules are oriented in different'directions at the two mica surfaces. The second, measured in both the planar and homeotropic orientations, is attributed to an enhanced order parameter near the surfaces. Both of these are monotonic repulsive forces measurable below $80 \mathrm{~nm}$. Finally, there is a short-range force which oscillates as a function of thickness, up to about six molecular layers, between attraction and repulsion. This results from ordering of the molecules in layers adjacent to the smooth solid surface. It is observed in both the planar and homeotropic orientations, and also in isotropic liquids.
\end{abstract}

1. Introduction. - It is generally accepted that when two solid bodies approach each other they interact via steric, electrostatic and electrodynamic (van der Waals) forces, but they may also interact indirectly,

(*) Present address : Physico-Chimie des Surtaces et des Membranes, C.N.R.S., U.E.R. Biomédicale, 45, rue des Saint-Pères, 75006 Paris, France. through forces mediated by a fluid between them. If there is any interaction between the solid and the molecules of the fluid, each solid surface has an effect on the structure of the fluid near it, and this effect propagates for some distance into the fluid. When two such surfaces come sufficiently close together that these boundary layers begin to overlap, the free energy of the system changes : it becomes a function 
of the distance between the surfaces, and so results in a force between the two solids. This type of indirect force has been variously called a structural force, solvent-mediated force, solvation force and - in aqueous media - hydration force [1].

Thus structural forces, as we shall call them, may arise when a solid interacts with a neighbouring fluid to modify its structure near the interface. Nematic liquid crystals are therefore good candidates for fluids in which to study such forces, since it is well known that solid boundaries affect the liquid crystal, if only by orienting it in a particular direction [2]. In this sense the familiar elastic forces observed in nematic liquid crystals may also be considered as structural forces. For example, two plates which impose fixed non-parallel orientations at their surfaces experience a repulsive force when they are brought together, due to the increasing energy of the splay, twist and bend modes which they cause in the liquid crystal between them.

A second type of structural force across a liquid crystal may arise if the surfaces affect the magnitude of the order parameter, i.e. the extent to which the elongated molecules are aligned, without disturbing the direction of alignment (which is given by a unit vector called the director). If a high degree of alignment is imposed at the surface, the order parameter decays back to its bulk value with a finite correlation length. Since an enhanced order parameter affects the free energy of the system (witness the modified nematicisotropic transition temperature in a thin film $[3,4]$ ), we may expect a structural force when two surfaces come within a few correlation lengths of each other.

Thirdly, boundaries may affect the positional ordering of the molecules. Consider for example a nematic liquid crystal whose molecules are aligned perpendicular to a smooth surface : it is easy to see that there will be a layer of molecules adjacent to the surface, then perhaps another layer next to the first, and so on for some distance - a positional correlation length into the liquid crystal. But this is just smectic ordering : the surface has had a very definite effect on the structure of the liquid crystal. This idea is more than just a hypothesis, for Manev et al. [5] and Rosenblatt and Amer [6] have observed exactly this effect. Manev et al. found that a free film of nematic $4{ }^{\prime}$-n-pentyl 4-cyanobiphenyl (5CB) between two air interfaces becomes smectic as the film gets very thin; Rosenblatt and Amer made a similar observation on 4-cyano benzylidene 4'-octyloxyaniline (CBOOA). As we shall see, this smectic ordering leads to a very special type of structural force.

In this paper we present the results of experiments in which all three of the above effects have been observed in 5CB at room temperature. The experiments employed a technique, not previously applied in liquid crystal studies, which is capable of measuring the force between two molecularly smooth solids as a function of their separation to a high degree of accu- racy. In addition, the technique allows the refractive index of the medium between the solids to be determined.

2. Experimental methods. - 2.1 ApPARATUS AND OPTICAL TECHNIQUE. - The apparatus and techniques have been described in detail elsewhere [7], and only a brief account of their operation is given here. A schematic drawing of the apparatus is shown in figure 1.

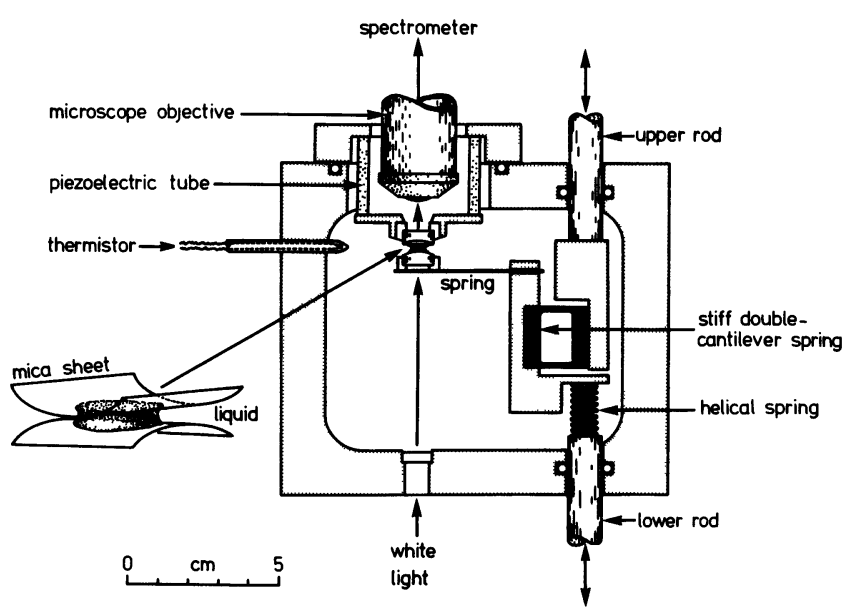

Fig. 1. - Schematic drawing of apparatus to measure forces between molecularly smooth surfaces. By use of white light and multiple beam interferometry the separation between the two surfaces can be measured to $\sim 0.1 \mathrm{~nm}$. The separation may be controlled by use of two micrometer-driven rods and a piezoelectric crystal to $\sim 0.1 \mathrm{~nm}$.

Some mica is cleaved to give a sheet which is molecularly smooth and of uniform thickness over a large area, and two pieces $(\sim 8 \mathrm{~mm} \times 8 \mathrm{~mm})$ are cut from this sheet. They are then glued to optically polished glass discs whose surfaces are cylindrical, and the discs are mounted with the axes of the cylinders at right angles as shown in the figure. The upper glass disc is rigidly attached to a piezoelectric crystal tube and the lower glass disc is mounted on a weak cantilever spring of known spring constant. The distance between the surfaces can be controlled coarsely (to $1 \mu \mathrm{m}$ ) by the upper micrometer-driven rod and finely (to $0.1 \mathrm{~nm}$ ) by means of (i) the lower micrometerdriven rod which moves the lower mica surface through a differential spring mechanism, and (ii) the piezoelectric crystal which moves the upper mica surface upon application of a voltage across the crystal walls. The piezoelectric tube expands or contracts by $\sim 0.7 \mathrm{~nm}$ $\mathrm{V}^{-1}$. These fine controls are readily calibrated by measuring how far they move the surfaces in the absence of any force, using the distance measuring technique described below.

Before gluing them to the glass discs, the back of 
each mica sheet is silvered to give a high reflectivity, so that there are sharp multiple-beam interference fringes between the silver layers. A grating spectrometer measures the set of wavelengths which is passed by this system, and these values can be used to calculate both the thickness and the refractive index of the medium between the mica surfaces [8]. Using this method the thickness can be measured to within 0.1-0.2 $\mathrm{nm}$. In addition, the shape of the fringes viewed in the spectrometer gives the mutual geometry of the two mica surfaces; thus the radii of curvature of the crossed cylindrical surfaces can be measured (typically $R \approx 1 \mathrm{~cm}$ ), and any surface deformations are immediately detected.

The interference fringes are split into two linearly polarized components by the birefringent mica. When the mica surfaces are in contact this gives the net birefringence of the two mica pieces, and thus their relative orientation, since they are of equal thickness and the maximum birefringence is known. When the mica surfaces are separated, the fringe splitting $\Delta \lambda / \lambda$ remains constant with an optically isotropic medium between them. However, with a birefringent medium such as a liquid crystal in the planar orientation, the splitting increases with sample thickness. Each polarization component can be measured independently to give the two refractive indices of the medium.

2.2 ForCe MEASUREMENT TECHNIQUE. - The force measurement technique involves displacing the base of the spring supporting the lower disc by a known amount relative to the upper surface, using either the previously calibrated lower micrometer-driven rod or the piezoelectric crystal (Fig. 1) while measuring the actual change in surface separation optically. Any difference in these two quantities, when multiplied by the spring stiffness $K$, gives the difference between the forces at the initial and final separations. In this way forces $F$ can be measured to $\pm 0.1 \mu \mathrm{N}$ at any separation $D$ down to contact.

An important aspect of the force measurement technique concerns the occurrence of instabilities : since one of the mica surfaces is suspended at the end of a spring of stiffness $K \approx 10^{2} \mathrm{~N} / \mathrm{m}$, forces can be measured only in regions where $\partial F / \partial D<K$. When the gradient of the force $\partial F / \partial D$ exceeds the stiffness $K$, instabilities occur leading to jumps (analogous to those occurring when two magnets, one of which is suspended from a spring, are brought towards each other). This matter is further discussed and illustrated in section 3.3.

2.3 Experimental PROCEDURE. - Most of the experiments were carried out with 5CB in its nematic phase at room temperature $\left(21-22^{\circ} \mathrm{C}\right)$, but additional clues about the nature of the forces were provided by experiments with the higher homologue 4 '-n-octyl 4-cyanobiphenyl (8CB), which is smectic-A at room temperature, and with $5 \mathrm{CB}$ above its nematic-iso- tropic transition temperature of $35^{\circ} \mathrm{C}$. The mica surfaces used were either freshly-cleaved, on which both 5CB and 8CB adopt the planar orientation, or coated with a monolayer of hexadecyltrimethylammonium bromide (HTAB) by the " retraction from solution " method [9], which gives a homeotropic orientation of both liquid crystals. To do this the mica was removed from a solution of $8 \times 10^{-4} \mathrm{M}$ HTAB in water, and dried, leaving behind a condensed monolayer $18 \AA$ thick on each surface. The thickness of the monolayers did not change when they came into contact with liquid crystal, nor did it vary in the course of an experiment. It was therefore assumed that HTAB did not dissolve in the liquid crystal.

Initially, the surfaces are brought into contact in air and the positions and shapes of the fringes at contact are measured to ascertain that there are no dust particles and that true molecular contact has been obtained. This contact defines $D=0$ (i.e. micamica contact for experiments in the planar orientation and HTAB monolayer-HTAB monolayer contact for the homeotropic case). The surfaces are then separated and a $0.1-0.3 \mathrm{ml}$ drop of liquid crystal is injected between them (see Fig. 1). The surfaces are now moved together and forces, distances, and refractive indices measured as described above.

\subsection{NOTE ON THE CROSSED-CYLINDER GEOMETRY} OF THE SURFACES. - It may be readily verified that the geometry of two cylindrical surfaces of radius $R$ positioned with their axes perpendicular to each other is equivalent to that of a sphere of radius $R$ near a flat surface (to leading order in $D / R$, which is $\lesssim 10^{-5}$ in these experiments). While this geometry may be less suitable for liquid crystal studies than that of two flat surfaces, there are two reasons why it is much more convenient. First, there are no problems in aligning the surfaces : for two flats it would be necessary to ensure that the two solid surfaces are not only smooth and flat to within $0.1 \mathrm{~nm}$, but that they are also aligned parallel to each other to within about $0.0005^{\circ}$, and maintained so during the movement of the surfaces.

The second reason is concerned with the interpretation of force measurements between two curved surfaces : in the present experiments the radius $R$ of the curved surfaces was $1-2 \mathrm{~cm}$, so that it was at least $10^{5}$ times greater than the range of distances, 0-80 nm, at which forces of interest were measured (equivalent to measuring the force between a sphere the size of the earth and a flat surface at distances from 0 to $60 \mathrm{~m}$ ). Thus locally the two curved surfaces appear almost indistinguishable from two flat parallel surfaces. For this situation Derjaguin [10] has shown that a simple integration of the force $F(D)$ between a sphere and a flat equals $2 \pi R \&(D)$ (provided $F(D)$ is of short range) where $\varepsilon(D)$ is the equivalent interaction energy between two flat surfaces per unit area at the same separation $D$. This relation

$$
\varepsilon=F / 2 \pi R
$$


is known as the " Derjaguin approximation » and has been found to hold for both electric double-layer forces and van der Waals forces [7]. Since the forces of interest in this study are of short range eq. (1) may be expected to be valid, and for this reason all forces $F$ will be plotted as $F / R$, i.e. normalized by $R$. We stress that this hypothesis plays no part in the actual measurements nor in the results shown, but only in how these are subsequently interpreted.

3. Results and interpretation. - The forces measured can be divided into three regimes which for convenience we term long-range, medium-range and short-range. We discuss each regime separately.

3.1 LONG-RANGE FORCES. - With a drop of liquid . crystal between the two mica surfaces, a force was measured at all separations from contact out to many microns, and it showed no signs of abating there. We ascribe this long-range force to the resolved components of the Laplace pressure in the drop and the surface tension acting at the mica-liquid crystal-air contact line [11]. For a constant drop volume, both of these change with distance in this geometry. Neither is of interest to us in this experiment.

The long-range force is rather irreproducible, presumably due to uneven wetting properties of the liquid crystal on the mica. This was observed regardless of whether or not the mica had been coated with HTAB. It persists when the sample is heated into the isotropic phase. Fortunately it varies rather slowly with distance, and shows no sign of « knowing » when the centres of the surfaces come near to or into contact $(D=0)$, so that over the comparatively short range of distance in which the other forces are measured $(0-80 \mathrm{~nm})$, the long-range force can be considered as a background or base-line force of constant slope $\partial F / \partial D$, and subtracted out. While this procedure is not difficult, it does lead to lower precision in the results for the forces discussed below than is usually achieved with this experimental technique.

3.2 Medium-RANGe FORCES. - 3.2.1 Planar alignment. - A monotonic repulsive force was measured at separations below about $80 \mathrm{~nm}$ : some typical results are shown in figure 2 . Generally there was some scatter in any given force run, but there was greater variability between different runs or experiments (with different mica surfaces being used in each experiment). One effect with which we could correlate this variability was that of forcing the two mica surfaces into contact : this often modified the force measured on subsequent runs.

The medium-range force vanished when the sample was heated well above the nematic-isotropic transition. Unfortunately, because the temperature control and measurement were not sufficiently precise, it was not possible to establish exactly how the force

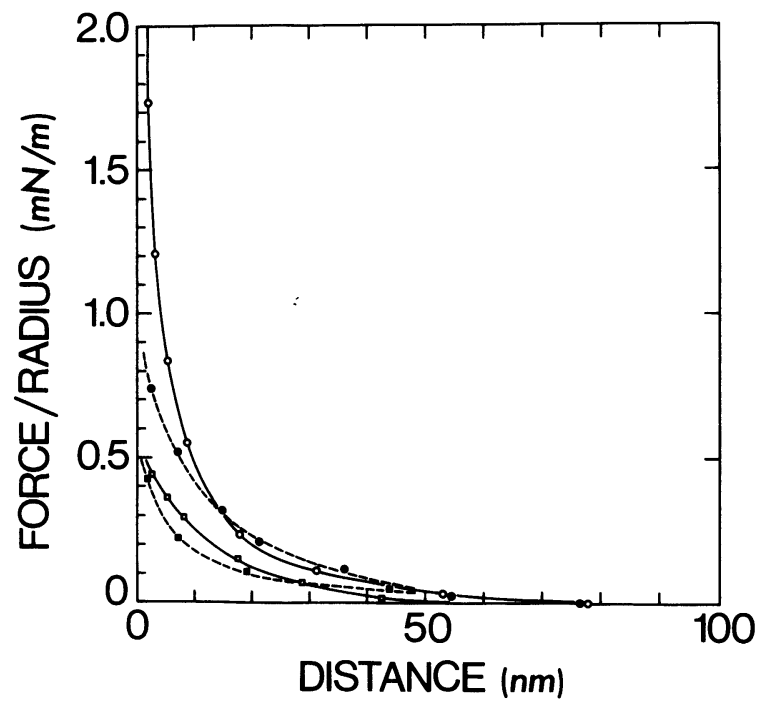

Fig. 2. - Typical repulsive medium-range force curves measured in $5 \mathrm{CB}$ in the planar orientation at $21-22^{\circ} \mathrm{C}$. A total of about forty force curves were measured, and all lay within the region covered by the four curves shown here. The angle of twist between the two surfaces was less than $10^{\circ}$.

decreased as a function of temperature in the region of the transition.

A reasonable assumption is that this force could be an elastic effect due to the nematic sample being twisted. Freshly-cleaved mica orients the 5CB molecules parallel to its surface, and presumably parallel to a particular crystallographic direction. If the two mica surfaces were mounted so that one was rotated with respect to the other, a twist would be imposed on the liquid crystal.

There are two ways in which we can investigate whether this accounts for the results observed. First, we can compare the results with a theoretical calculation of the force expected in a twisted sample, and second, we can vary the angle of twist in an experiment to see how this affects the force.

Taking the first approach, let us consider the elastic energy stored in the liquid crystal between the two mica surfaces. The geometry, which as mentioned in section 2.4 is equivalent to that of a sphere near a flat plate, makes an exact calculation of the elastic energy extremely difficult. Nevertheless, if we are allowed some approximations, we can estimate it. First consider the case of a nematic liquid crystal in the planar orientation between two parallel flat plates a distance $t$ apart, but with the alignment direction of one plate rotated by an angle $\theta_{\mathrm{t}}$ with respect to the other. There is then a uniform twist imposed on the nematic, and the corresponding elastic energy per unit area of the plates is [2]

$$
\varepsilon_{\mathrm{T}}=\frac{K_{22}}{2} \frac{\theta_{\mathrm{t}}^{2}}{t}
$$

where $K_{22}$ is the Frank twist elastic constant. 
To obtain the force between a sphere of radius $R$ and a flat plate a distance $D$ from it (see Fig. 3) we make the following approximations :

(i) We assume the director is everywhere parallel to the flat plate, whereas in practice, in the vicinity of a curved surface it will be parallel to that surface. The error involved in this assumption is small, since the angle between the director at the upper surface and the horizontal plane is $\varphi$ (see Fig. 3), which only becomes significant as $d$ increases, but as this happens the energy $\varepsilon \propto \frac{1}{D+d}$ decreases. This point will be discussed further in section 3.2.2.

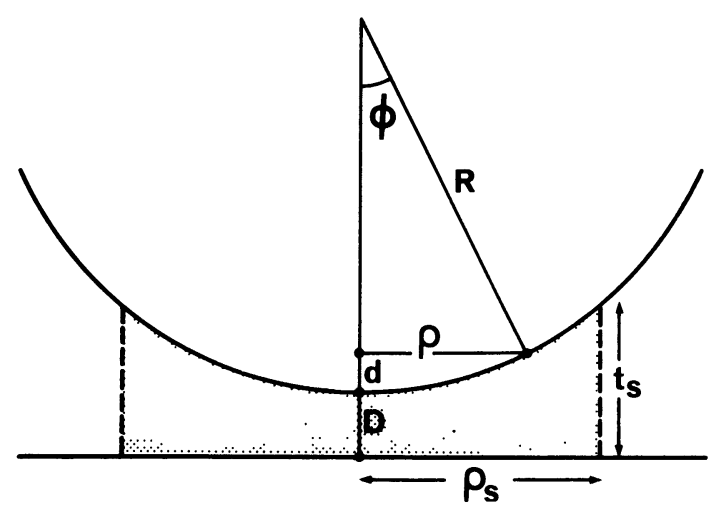

Fig. 3. - The geometry of a sphere near a flat plate, showing the symbols used in the discussion of elastic forces.

(ii) We ignore variations in the director along horizontal directions, since these are much more gradual than those in the vertical direction.

(iii) We assume that at a given position in the sample the twist is uniform across the thickness of the sample at that position, so that the energy is given by (2).

(iv) We ignore distortions of the orientation at the meniscus bounding the liquid crystal drop.

With these assumptions, the twist energy per unit area at a radius $\rho$ is

$$
\varepsilon_{\mathrm{T}}=\frac{K_{22}}{2} \frac{\theta_{\mathrm{t}}^{2}}{(D+d)} .
$$

Integrating out to the edge of the drop at $\rho_{\mathrm{s}}$ gives the total twist energy

$$
E_{\mathrm{T}}=\int_{0}^{\rho_{\mathrm{s}}} 2 \pi \rho \mathrm{d} \rho \frac{K_{22}}{2} \frac{\theta_{\mathrm{t}}^{2}}{(D+d)} .
$$

Changing the integration variable to the sample thickness

$$
t=D+d=D+R-\sqrt{R^{2}-\rho^{2}},
$$

so

$$
\mathrm{d} t=\frac{\rho \mathrm{d} \rho}{(D+R-t)},
$$

gives

$$
\begin{aligned}
E_{\mathrm{T}} & =\pi K_{22} \theta_{\mathrm{t}}^{2} \int_{D}^{t_{\mathrm{s}}}\left(\frac{D+R-t}{t}\right) \mathrm{d} t \\
& =\pi K_{22} \theta_{\mathrm{t}}^{2}\left((D+R) \log \frac{t_{\mathrm{s}}}{D}+D-t_{\mathrm{s}}\right),
\end{aligned}
$$

where $t_{\mathrm{s}}$ is the sample thickness at the edge of the drop, and is typically $\sim 1 \mathrm{~mm}$.

The force between the two surfaces is

$$
\begin{aligned}
F_{\mathrm{T}} & =-\frac{\partial E_{\mathrm{T}}}{\partial D} \\
& =\pi K_{22} \theta_{\mathrm{t}}^{2}\left(\frac{R}{D}-\log \frac{t_{\mathrm{s}}}{D}-\frac{\left(D+R-t_{\mathrm{s}}\right)}{t_{\mathrm{s}}} \frac{\partial t_{\mathrm{s}}}{\partial D}\right) \\
& \simeq \pi K_{22} \theta_{\mathrm{t}}^{2} \frac{R}{D},
\end{aligned}
$$

since $D \ll t_{\mathrm{s}} \ll R$ in our experiments, and for constant drop volume $\partial t_{\mathrm{s}} \partial D \approx-\tan ^{2} \varphi_{\mathrm{s}} \approx-0.2$. Thus

$$
\frac{F_{\mathrm{T}}}{R}=\frac{\pi K_{22} \theta_{\mathrm{t}}^{2}}{D}=2 \pi \varepsilon_{\mathrm{T}},
$$

as expected from the Derjaguin approximation (1). Indeed, all we have done is work through an example where the Derjaguin approximation is expected to hold because the force falls off sufficiently rapidly with distance (i.e. the range of the force is much less than $t_{\mathrm{s}}$ ).

Is this force large enough to explain the experimental results shown in figure 2 ? As mentioned in section 2.1, the measured birefringence of the two mica sheets in contact gives us their relative orientation. From this we can establish that in the experiments whose results are presented in figure 2, the two micas were oriented parallel to within $10^{\circ}$. Putting the maximum possible twist $\theta_{\mathrm{t}}=10^{\circ}$ in equation (5), and using

$$
K_{22}=6.5 \times 10^{-12} \mathrm{~N}
$$

for $5 \mathrm{CB}$ at $22{ }^{\circ} \mathrm{C}$ [12], we find $\left(F_{\mathrm{T}} / R\right)_{\max } \simeq 0.06 \mathrm{mN} / \mathrm{m}$ at $D=10 \mathrm{~nm}$, which is too small to match the experimental range of values of $0.2-0.5 \mathrm{mN} / \mathrm{m}$.

The second method of investigating if these forces are due to twist is to vary the twist during an experiment and see whether the forces are modified. The results of such an experiment are shown in figure 4. Initially the mica surfaces had their optic axes parallel, and the force curve labelled $\theta_{t}=0$ was measured. The glass disc carrying one of the mica sheets was then rotated by $15^{\circ}$, and the second curve was measured; the third curve was measured after a $33^{\circ}$ rotation. Finally, the mica surface was rotated back to its original position, and a curve very close to the first (lowest) curve was measured.

In rotating one glass disc through an angle $\theta_{t}$ we also altered the crossed cylindrical geometry. It can be shown (D. Y. C. Chan, personal communication) 


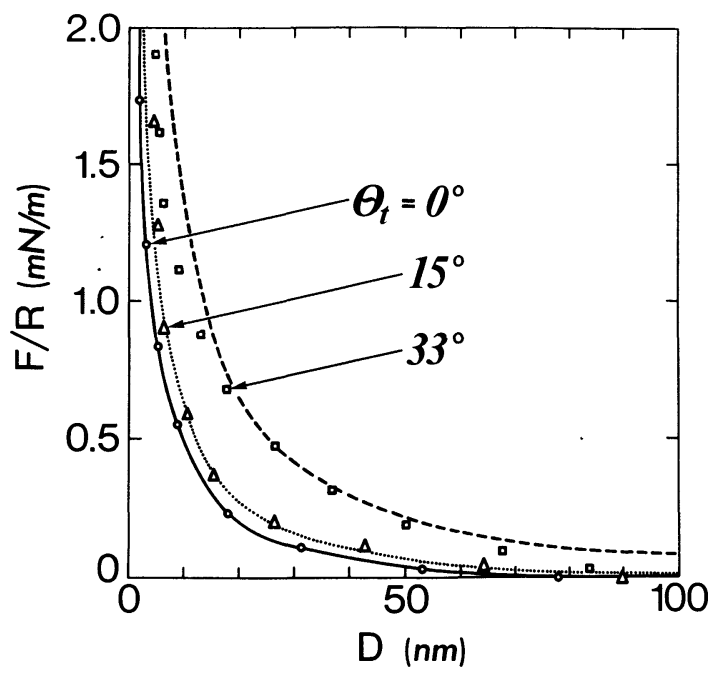

Fig. 4. - Medium-range forces for 5CB in the planar orientation between two mica surfaces, one of which is rotated by an angle $\theta=0^{\circ}(O), 15^{\circ}(\triangle)$ and $33^{\circ}(\square)$ with respect to the other, so that a twist is imposed on the sample. The continuous line is the baseline curve drawn through the $\theta=0^{\circ}$ points ; the dotted and dashed lines are obtained by adding to this curve the theoretical twist force calculated from equation (6) for $\theta=15^{\circ}$ and $\theta=33^{\circ}$ respectively.

that this increases the force between the cylinders by a factor $1 / \cos \theta_{\mathfrak{t}}$, so equation (5) becomes

$$
\frac{F_{\mathrm{T}}}{R} \text { (skew cylinders) }=\frac{\pi K_{22} \theta_{\mathrm{t}}^{2}}{D \cos \theta_{\mathrm{t}}} .
$$

Since the force does not vanish at $\theta_{\mathrm{t}}=0^{\circ}$, it appears that this curve results from some other effect. Supposing this to be so, i.e. considering it as a base line to which the twist force is added, we can then account for the results reasonably well using equation (6) : it is apparent that the first rotation of $15^{\circ}$ has only a small effect on the force, but rotation by a further $18^{\circ}$ has a much larger effect, in accordance with a force proportional to $\theta_{t}^{2} / \cos \theta_{\mathrm{t}}$. Putting $K_{22}=6.5 \times 10^{-12} \mathrm{~N}$ [12] into equation (6) and adding the resulting theoretical twist force $F_{\mathrm{T}} / R$ to the experimental base-line curve $\theta_{t}=0$ (solid line) in figure 4 gives the dotted curve for $\theta_{t}=15^{\circ}$ and the dashed curve for $\theta_{t}=33^{\circ}$. The agreement with experiment is then good for $\theta_{t}=15^{\circ}$, and while it is not so good for $\theta_{t}=33^{\circ}$, the magnitudes of the calculated twist forces still appear to be about right to explain the results if they are added to the base curve. Equation (6) alone is not enough to account for the results.

We therefore conclude that more than one type of force is acting in this region : an elastic force if there is twist in the sample; plus some other component which is present with or without twist. At the moment this component has not been explained; a hypothesis about its origin is given in section 3.2.3.

It is appropriate at this point to discuss the strength of the liquid crystal anchoring on mica, and to report an interesting observation. First, how strong is the tendency for the 5CB molecules to be oriented in a particular direction parallel to the mica surface, and is it strong enough to maintain this orientation when another surface, with a different orientation, comes very close to it and creates a highly twisted sample?

Because the liquid crystal does have a preferred orientation on the mica, we may suppose that the surface energy of the mica-5CB interface depends on orientation, and has a minimum in the preferred direction. Thus the surface energy per unit area may be expressed as

$$
W=W_{0}+W_{s}(\theta),
$$

where $\theta$ is the angle between the alignment direction and the most favourable direction; the angle-dependent anchoring energy $W_{\mathrm{s}}$ vanishes at $\theta=0$ or $\pi$, and is positive for all other angles. As two surfaces approach, the twist energy in the liquid crystal may be reduced at the expense of the surface energy if the anchoring at one or both surfaces changes. The twist energy at the thickness where this occurs should give a measure of $W_{\mathrm{s}}$.

In our experiments, the liquid crystal was never observed to untwist as the mica surfaces were brought together, suggesting that the anchoring energy is large. However, in the experiment where $\theta_{t}$ was set to $33^{\circ}$, we discovered that the sample was untwisted after the surfaces were forced into molecular contact. It then remained untwisted (in the central region of the curved surfaces) on separating the surfaces, until at a separation $D=830 \pm 10 \mathrm{~nm}$ the twisted configuration was suddenly restored. Repeating this several times confirmed that the sample was only untwisted when it was forced into contact, and that the twist always returned abruptly at the same comparatively large separation.

At $D=830 \mathrm{~nm}$ the twist energy per unit area of surface is given by equation (2) as $1.3 \times 10^{-3} \mathrm{~mJ} / \mathrm{m}^{2}$ $\left(\mathrm{erg} / \mathrm{cm}^{2}\right)$. Assuming the orientation changed at only one surface, this suggests that

$$
W_{\mathrm{s}}\left(33^{\circ}\right)=1.3 \times 10^{-3} \mathrm{~mJ} / \mathrm{m}^{2},
$$

which, while small, is typical for the anchoring energy of liquid crystals on solid substrates [13]. However, there must be a large energy barrier to changing the orientation from its preferred direction to explain the hysteretic behaviour described above. Since on approach the liquid crystal did not unwind until $D \lesssim 1 \mathrm{~nm}$, the energy barrier must be at least $10^{3}$ times higher than the energy difference $W_{\mathrm{s}}$. There appears to be little or no barrier to returning to the most favourable surface orientation.

We may also note that the form for $W_{\mathrm{s}}$ suggested by Meyer [14] as the simplest function having the correct dependence on $\theta$,

$$
W_{\mathrm{s}}=A \sin ^{2} \theta,
$$


is too " soft " to explain the sudden transition from an untwisted to a twisted sample which we observed. If $W_{\mathrm{s}}$ had the form (8) the transition would be more gradual, and the twist angle would increase steadily from zero at contact to its full value at very large separations.

3.2.2 Homeotropic orientation. - With the nematic liquid crystal oriented perpendicular to mica surfaces coated with a HTAB monolayer, a medium-range force was also measured, but it was always very weak - similar to the lowest curve shown in figure 2. It is not shown separately. This force was only measured beyond $D \simeq 10 \mathrm{~nm}$; closer than that the force was dominated by the short-range effects to be discussed in section 3.3.

Again, this force cannot be attributed to elastic forces, because in this orientation the elastic energies are extremely small. If first we consider a nematic liquid crystal between two parallel flat surfaces with the director perpendicular to one surface and making an angle $\varphi_{\mathrm{t}}$ to the normal at the other, there is a uniform splay-bend distortion of the liquid crystal whose energy per unit area of surface can be calculated as

$$
\varepsilon_{\mathrm{SB}}=\frac{1}{2} \frac{\varphi_{\mathrm{t}}^{2}}{t}\left\{\frac{1}{2}\left(K_{11}+K_{33}\right)+\frac{\sin 2 \varphi_{\mathrm{t}}}{4 \varphi_{\mathrm{t}}}\left(K_{33}-K_{11}\right)\right\} \text {, }
$$

where $t$ is the distance between the surfaces. This has the same form as the twist energy (2), with an effective elastic constant

$$
\bar{K}=\frac{1}{2}\left(K_{11}+K_{33}\right)+\frac{\sin 2 \varphi_{\mathrm{t}}}{4 \varphi_{\mathrm{t}}}\left(K_{33}-K_{11}\right) .
$$

For small $\varphi_{\mathrm{t}}$ this reduces to the bend elastic constant $K_{33}$.

Between curved surfaces, where the director is always normal to each surface, the situation differs from the twist case considered earlier because now the angle $\varphi_{\mathrm{t}}$ is determined by the geometry of the surface (Fig. 3). In the thinnest part of the sample the surfaces are nearly parallel so there is hardly any elastic distortion; only in the thicker regions does the angle between the surfaces become significant, but then the elastic energy is small because it decreases with thickness. In fact (see Fig. 3)

$$
\cos \varphi_{\mathrm{t}}=\frac{R-d}{R},
$$

so to leading order

$$
\varphi_{\mathrm{t}}^{2}=\frac{2 d}{R} .
$$

Thus from equation (9), when $D=0, t=d$ and

$$
\varepsilon_{\mathrm{SB}} \simeq \frac{K_{33}}{R}=\text { constant }
$$

i.e. $\varepsilon_{\mathrm{SB}}$ is independent of $d$ throughout the sample. Thus the splay-bend elastic energy per unit area does not fall off with thickness in this geometry because $\varphi_{\mathrm{t}}^{2}$ increases linearly with $t$, and the Derjaguin approximation cannot be made. We remark that at $D=0$, where the energy is greatest, it is still rather small :

$$
\frac{K_{33}}{R} \sim 10^{-6} \mathrm{mN} / \mathrm{m} \text {. }
$$

(We expect this energy to be comparable to the energy we neglected in making the first approximation in section 3.2.1 by supposing that the director in the twisted planar sample always remained parallel to only one of the surfaces.)

The observation that the Derjaguin approximation is invalid here does not prevent us from integrating to find the total energy between the surfaces, then differentiating with respect to separation to find the elastic force, if we are allowed to make approximations similar to those made in the twist case. Thus if we assume that variations of the director in horizontal directions are much slower than in the vertical, that the director varies uniformly with sample thickness at any position, and that distortions at the meniscus are neglected, we find that the splay-bend elastic force is

$$
\frac{F_{\mathrm{SB}}}{R} \simeq \frac{2 \pi K_{33}}{R} \log \frac{t_{\mathrm{s}}}{D},
$$

where $t_{\mathrm{s}}$ is the thickness at the edge of the sample. Using $K_{33}=1.76 \times 10^{-11} \mathrm{~N}$ [12] and typical experimental values $t_{\mathrm{s}}=1 \mathrm{~mm}$ and $R=1 \mathrm{~cm}$ gives

$$
\frac{F_{\mathrm{SB}}}{R}=1.3 \times 10^{-4} \mathrm{mN} / \mathrm{m}
$$

at $D=10 \mathrm{~nm}$ : this force is indeed much too weak to match the experimental results.

As in the planar case, we are left with a force, albeit small, which is not accounted for by classical elastic effects. We will now investigate whether it can be correlated with the degree (not direction) of alignment near the surfaces. The degree of alignment is given by the conventional nematic order parameter $S$, which can be obtained from measurements of the refractive indices.

3.2.3 Refractive indices of very thin nematic films. The optical technique employed in these experiments [8] allows us to measure not only the thickness of the liquid film but also its refractive index, averaged across the film. With the nematic liquid crystal in the planar orientation both refractive indices $n_{\mathrm{e}}$ and $n_{0}$ are measurable; in the homeotropic orientation only the ordinary index $n_{0}$ is obtained.

Figure $5 a$ shows the refractive indices $n_{\mathrm{e}}$ and $n_{0}$ measured in thin films of nematic $5 \mathrm{CB}$ at $21-22^{\circ} \mathrm{C}$; the measurements were made around $\lambda=550 \mathrm{~nm}$. The hollow circles show results obtained in the planar orientation $\left(n_{0}\right.$ and $\left.n_{\mathrm{e}}\right)$, and the filled circles are those 

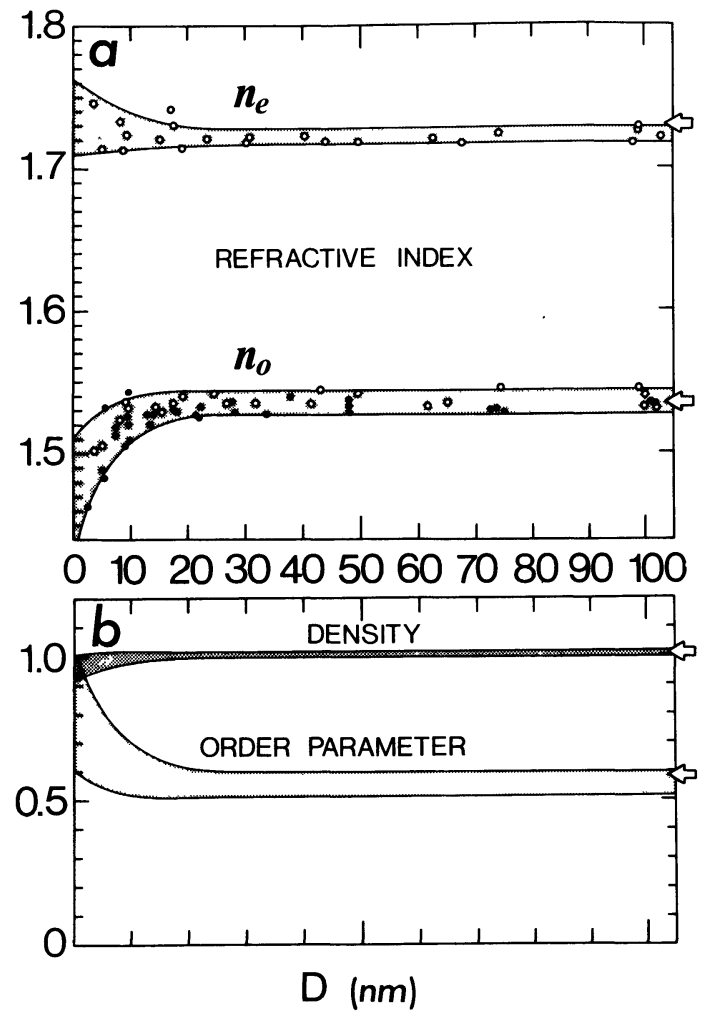

Fig. 5. - a) Refractive indices $n_{\mathrm{e}}$ and $n_{0}$ of a thin film of 5CB at room temperature $\left(21-22^{\circ} \mathrm{C}\right)$ plotted as a function of film thickness. Results are from a number of experiments, in both the planar $(\mathrm{O})$ and homeotropic (O) orientations. Shaded regions indicate confidence limits within which most of the measurements lie. The bulk values [15] are indicated by arrows on the right-hand axis. b) Order parameter and density (in $\mathrm{g} / \mathrm{cm}^{3}$ ) calculated from the refractive index measurements according to equations (15) and (16). Arrows on the right-hand axis indicate the bulk values.

obtained in the homeotropic ( $n_{0}$ only). The values of $n_{0}$ obtained from both orientations agree reasonably well; if anything the homeotropic measurements lie somewhat below the planar ones, especially near contact.

At film thicknesses greater than $30 \mathrm{~nm}$ these measurements agree well with bulk values [15] marked by arrows on the right-hand axis. As the thickness decreases $n_{\mathrm{e}}$ appears to increase and $n_{0}$ to decrease, indicating that the birefringence $n_{\mathrm{e}}-n_{0}$ increases. Unfortunately the determination of the refractive indices becomes less accurate for small film thicknesses. The shaded regions in figure $5 a$ mark « confidence limits » bounding almost all of the experimental points.

In principle both the order parameter $S$ and the density $\rho$ can be calculated from the two refractive indices. While the calculation does involve some rather drastic assumptions, it gives results which are reasonable, and certainly adequate for our purposes of investigating how $S$ and $\rho$ change near a solid surface. As shown by Horn [15],

$$
S=\frac{\bar{\alpha}}{\alpha_{\|}-\alpha_{\perp}}\left(\frac{n_{\mathrm{e}}^{2}-n_{0}^{2}}{\bar{n}^{2}-1}\right)
$$

and

$$
\rho=\frac{M}{N_{\mathrm{A}}} \cdot \frac{3}{4 \pi \bar{\alpha}}\left(\frac{\bar{n}^{2}-1}{\bar{n}^{2}+2}\right),
$$

where $\bar{n}^{2}=\frac{1}{3}\left(n_{\mathrm{e}}^{2}+2 n_{0}^{2}\right), \alpha_{\|}$and $\alpha_{\perp}$ are the molecular polarizabilities parallel and perpendicular to the long axis of the molecule, $\bar{\alpha}=\frac{1}{3}\left(\alpha_{\|}+2 \alpha_{\perp}\right), M$ is the molecular weight and $N_{\mathrm{A}}$ is Avogadro's number.

The order parameter and density were calculated taking $n_{\mathrm{e}}$ and $n_{0}$ from the limiting values of the shaded regions in figure $5 a$, and using the values

$$
\bar{\alpha}=3.36 \times 10^{-29} \mathrm{~m}^{3} \text { and } \frac{\alpha_{\|}-\alpha_{\perp}}{\bar{\alpha}}=0.70
$$

for $\lambda=550 \mathrm{~nm}$ [15]. Results are shown by the corresponding shaded regions in figure $5 b$, with the bulk values indicated by the arrows on the right-hand axis. The order parameter is enhanced in very thin films of 5CB between mica surfaces, whereas the density may be decreased slightly.

If the mica surfaces act to increase the order parameter of the liquid crystal near them, it is somewhat surprising that they should decrease its density : one would expect that if anything the density would be increased. If we examine figure 5 more closely we see that in fact the apparent small decrease in density may be an artefact of the way in which we have calculated it. The lower limit of density is calculated from the lower limits of $n_{\mathrm{e}}$ and $n_{0}$, but close inspection of figure $5 a$ shows that the lower limit of $n_{0}$ is determined by values measured in the homeotropic orientation, for which values of $n_{\mathrm{e}}$ cannot be obtained. It could be that in this orientation $n_{\mathrm{e}}$ is also correspondingly higher, so that the density is constant or slightly increased, and the order parameter (averaged across the film) is higher than in the planar case at the same thickness. This is in accord with the result of Schröder [4] that an enhanced order parameter at the surface of a liquid crystal has a longer coherence length in the homeotropic orientation than in the planar.

In a very thin film in the planar orientation the liquid crystal is likely to be biaxial. There are two possible reasons for this : one is that the thermal fluctuations of the molecules are restricted, so that they can rock freely in a plane parallel to the surface but are hindered from rocking in a plane perpendicular to the surface. The second is that rotation of the molecules - which do not have complete cylindrical symmetry - may be restricted for those molecules closest to the surface. In section 3.3.3 we find some evidence for this. However, in either case the biaxiality is unlikely to be detected by our measurements of birefringence, especially since they are least accurate in the thinnest films.

Can the effect of the mica surfaces, in increasing the degree of alignment of the molecules in the neighbouring nematic liquid crystal, lead to a structural force ? 
This question is in fact a very general one, pertinent to any liquid medium in which a change in some order parameter reflects the degree of surface-induced structural changes. Full calculations of structural forces have only been made on specific model systems $[1,16-19]$.

The total free energy of the solid-liquid-solid system is determined by three types of interaction : the solidsolid, liquid-liquid and solid-liquid interactions. In our case the direct solid-solid interaction is just the van der Waals attraction between two pieces of mica separated by a liquid crystal; its magnitude is much smaller than the forces shown in figure 2 . It is the other two terms which determine the structural force.

The liquid-liquid contribution can be handled by making a Landau expansion of the free energy in powers of the order parameter and its gradients, as first suggested by Marčelja and Radic [20]. In the homeotropic orientation the liquid crystal is always uniaxial and the degree of alignment is described by a scalar order parameter, whereas in the planar orientation the alignment may be biaxial, as described above, in which case the order parameter is a tensor with two non-zero components. Because the order parameter is modified near the surfaces, the free energy of the liquid itself is higher than it would be in the bulk; what we need to know is how it changes with separation between the surfaces. The theory of Marčelja and Radić [20] predicts that in our case where the order parameter profile across the liquid crystal film is symmetric, the free energy from this contribution decreases as the surfaces approach, i.e. it leads to an attraction.

However, Marčelja and Radić do not consider the final term, the solid-liquid interaction, which is what causes the order parameter to be modified in the first place. Without a detailed model of the interactions involved it is not possible to say whether this contribution to the free energy increases or decreases with separation, nor how its magnitude compares with the liquid-liquid term. In order to account for our results by this explanation, the solid-liquid term must give a repulsion which is large enough to overcome the Marčelja-Radić attractive term.

We postulate that both the medium-range repulsive forces measured in the planar orientation (Fig. 2) and similar forces measured in the homeotropic orientation (not shown) are due to the order parameter effects we have discussed. However, in the absence of a plausible theory which accounts for the sign, magnitude and range of these forces, we cannot be fully certain of our ascription.

3. 3 SHORT-RANGE FORCES. - When the two mica surfaces come very close to contact, so that the liquid crystal film is less than about six molecules thick, the interaction becomes dominated by a completely different force. This is seen in both the planar and homeotropic orientations. We discuss the homeotropic case first.
3.3.1 Homeotropic orientation. - For D less than about $15 \mathrm{~nm}$ the force no longer varies monotonically but becomes oscillatory, that is, it alternates as a function of separation between attraction and repulsion. This is seen in figure 6 , where we have plotted the force at very short range in the homeotropic orientation. The amplitude of these spatial oscillations increases as the surfaces come closer, with a final steep drop into an attractive well (adhesion) when the surfaces come into molecular contact between their HTAB monolayers. The monotonic medium-range forces discussed in the previous section are too small to be resolved on the scale of figure 6 .

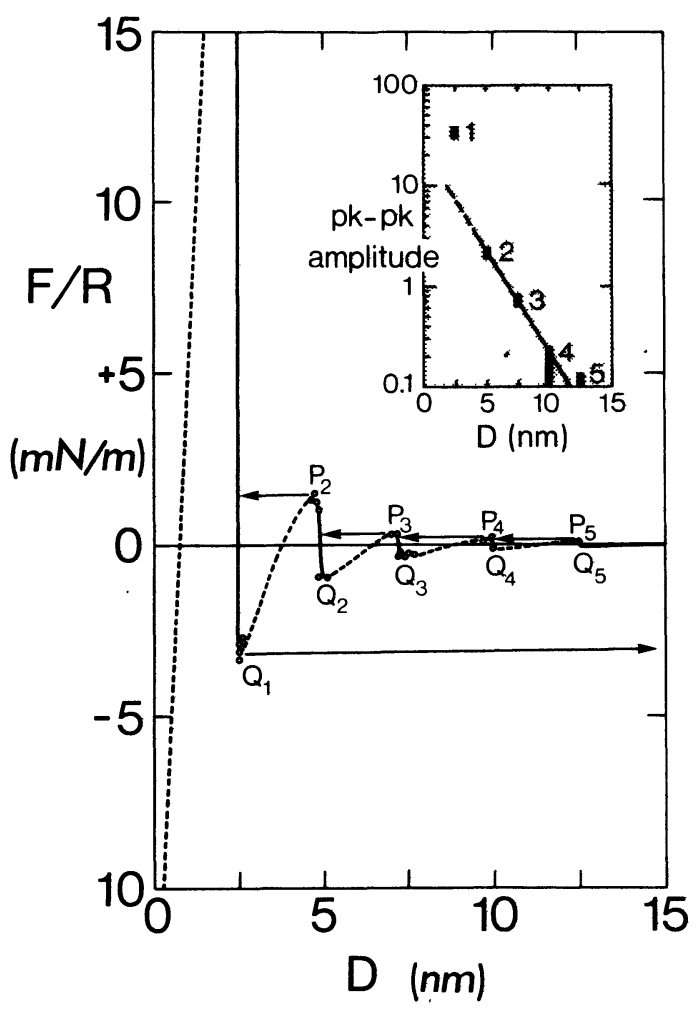

Fig. 6. - Short-range force for 5CB in the homeotropic orientation. Points $\mathbf{P}$ and $\mathrm{Q}$ represent force maxima and minima, respectively, from which surfaces jump in or out as described in the text. $D=0$ corresponds to contact between the two HTAB monolayers, whose thickness was observed to remain constant throughout an experiment. The dashed sections of the curve represent experimentally inaccessible (unstable) force regimes. The inset shows the peak-topeak amplitudes, P-Q, on a log plot. The exponential decay length is about $2.2 \mathrm{~nm}$.

In section 2.2 it was pointed out that with the spring deflection method of measuring forces, the system is unstable in regions where $\partial F / \partial D>K$, where $K$ is the stiffness of the spring supporting one of the mica surfaces. These unstable regions are shown by the dashed sections of the curve in figure 6. It is important to note that because these sections are experimentally inaccessible (without the use of a much stiffer spring, which would reduce the measuring sensitivity) we cannot determine their shape. The dashed lines in figure 6 are drawn fairly straight for simplicity, but 
in fact there is no reason to believe that the real force curve has the saw-tooth form we have drawn. Indeed, as we will discuss shortly, there is reason to believe that the shape is quite different. We do know, however, that the stable regions marked by continuous lines have the correct form : the force increases very steeply at certain discrete separations, and it is unusually difficult to move the surfaces from these separations.

When the surfaces are brought towards each other in an experiment, once they reach an unstable region there is a jump inwards to the next stable region. These jumps occur from the points labelled $P$ in figure 6 , and are indicated by the arrows, which have slope

$$
\frac{\partial(F / R)}{\partial D}=\frac{K}{R} \approx 10^{4} \mathrm{~N} \cdot \mathrm{m}^{-2}=10^{-2} \mathrm{mN} / \mathrm{m} \cdot \mathrm{nm}^{-1} .
$$

On separating the surfaces, there are jumps outwards from the force minima labelled $Q$. In this case the surfaces usually "jump over» the other minima, and only come to rest at large distances $D$ where $F / R \simeq 0$. The observation of such jumps shows that the force law is an oscillatory function of distance $D$. By measuring the start and end points of the jumps we determine both the positions and the magnitudes of the force maxima and minima.

We attribute this force curve to a strong tendency of the 5CB molecules to arrange themselves in smectic layers when sandwiched between two molecularly smooth mica surfaces. Our results are in full accord with the very different experiments of Leadbetter et al. [21] who showed from X-ray diffraction measurements that in bulk 5CB there are local regions in which the molecules have smectic ordering. The layer spacing is $2.57 \mathrm{~nm}$ at $17^{\circ} \mathrm{C}$ (and should be smaller at higher temperatures), and the smectic correlation extends for four or five layers. From figure 6 we see that the periodicity of the force oscillations is $2.5 \mathrm{~nm}$. Our results also agree with those of Manev et al. [5], who observed that there is smectic ordering in thin films of 5CB between air interfaces when the film thickness is reduced below $25 \mathrm{~nm}$, with a layer spacing of $2.5 \mathrm{~nm}$. The fact that Manev et al. observed discrete layers at film thicknesses greater than ours suggests that their experiment provides a more sensitive way of detecting smectic ordering.

If one accepts that the Derjaguin approximation is valid for this short-range force, then the oscillatory function shown in figure 6 is easy to interpret as ( $2 \pi$ times) the free energy per unit area of a homeotropic liquid crystal film of thickness $D$ between two flat plates. There are energy minima when $D$ corresponds to an integral number of smectic layers, but the energy rises sharply at intermediate distances where the layers must be either expanded or compressed. This is most pronounced in the thinnest films; as the number of layers increases it becomes less expensive energetically to accommodate a non-integral number of layers. The surfaces only impose coherent smectic ordering on a region of sample adjacent to them apparently for about three smectic layers - so for thicker films at least part of the film is nematic, and the energy varies monotonically.

The derivation of the Derjaguin approximation [10] is valid so long as $F(D)$ is mathematically well behaved, i.e. is single-valued and differentiable. These criteria are satisfied by the curve shown in figure 6, even though sections of it are very steep. Physically, if the liquid crystal has smectic layers, one would expect circular edge dislocation loops in this geometry. However, the « dislocations " here probably bear no resemblance to dislocations in a bulk smectic, because layers only one or two layers away from the "dislocation " are forced by the mica surfaces to be (locally) planar. This is contrary to the arrangement adopted by layers near a dislocation in bulk [22], and would have the effect of spreading the " core region " along the plane of the layers to such an extent that the concept of a dislocation line is no longer applicable. As we move out from the centre of the surfaces, alternate regions of the sample would be in compression and extension, and their contributions to the force would tend to cancel. In addition, they diminish as the sample thickness increases away from the centre; the most important contribution comes from the comparatively flat central region.

The tendency of the sample to form an integral number of layers is so strong that in very thin films the liquid crystal can flatten the curved surfaces. This is achieved by deforming the glue holding the mica to the glass, and leads to a noticeable change in shape of the interference fringes when there are only one or two smectic layers between the surfaces. When this happens the local radius of curvature is increased, so that the values of $F / R$ shown in figure 6 , calculated assuming a constant radius $R$, should be reduced at small $D$ : in particular the first peak is probably not as high as shown.

The inset in figure 6 shows the peak-peak height of the oscillations (measured for the same number of layers, thus $P_{1}-Q_{1}, P_{2}-Q_{2}$, etc.) plotted on a logarithmic scale as a function of $D$. It appears that the amplitude decays roughly exponentially with distance, and while we do not suggest that this has any theoretical significance, it does enable us to define a decay length giving a measure of the persistence of the oscillations in the force curve. In this case the decay length is $2.2 \mathrm{~nm}$, close to the layer spacing of $2.5 \mathrm{~nm}$.

Figure 7 shows the force-distance curve measured in a different experiment. The points measured on bringing the surfaces towards each other are shown as filled circles and those measured on separation are shown as open circles. Force minima were also detected at about 15.0 and $17.5 \mathrm{~nm}$, but these were too weak to be measured accurately. In this case there are still strong oscillations in the curve, although the force is now overall repulsive. Thus the 5CB molecules form smectic layers adjacent to the surface even when the 


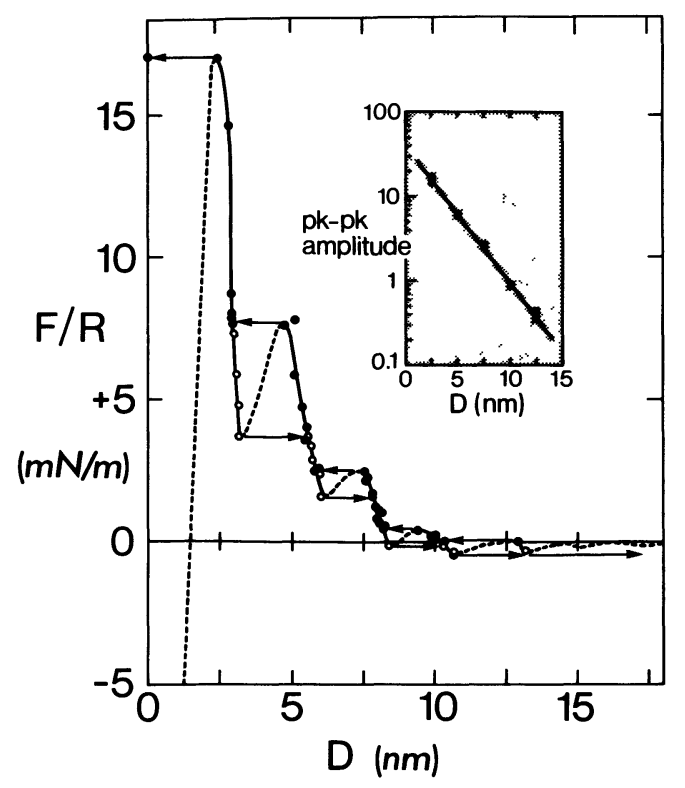

Fig. 7. - Short-range force for 5CB in the homeotropic orientation, with suspected roughness in HTAB surfaces (see text). Closed circles represent the forces measured on approach of the surfaces; open circles on separating the surfaces. The exponential decay length of the peak-to-peak amplitude is $2.65 \pm 0.20 \mathrm{~nm}$ (inset).

overall force is quite different, due probably to a slight roughness of the HTAB monolayer (although the presence of surface-active impurities cannot be ruled out as an explanation).

The differences between this force curve and that shown in figure 6 are : first, it is repulsive over most of its range; second, the oscillations are « softer ", that is, the intervals of distance $\Delta D$ in which the system is stable are wider - typically $1.0 \mathrm{~nm}$ here - whereas in figure 6 the system is only stable for a compression or expansion of about $0.1 \mathrm{~nm}$ per layer. According to the following argument, both of these differences could be ascribed to some roughness of the adsorbed HTAB monolayer. Suppose that the monolayer was not densely packed in this experiment, and there were surface asperities of $\Delta D \sim 1 \mathrm{~nm}$, so that the smectic layers mimicked this roughness. The measured force would then be an average of the forces at all separations spread over a $1 \mathrm{~nm}$ " window "; in other words, the force curve would be a convolution of the ideal curve for perfectly smooth surfaces with a « roughness function " reflecting the distribution of heights of the surface. Such a convolution would certainly « soften » the steep dips in the force curve; it would also give an overall repulsion if the ideal force curve was repulsive at most distances, i.e. if the attractive wells, although deep, were very narrow (so that the integral of the force curve was positive). Physically, this seems a reasonable supposition for the shape of the ideal curve : any deviation from an integral number of layers is extremely difficult. Thus the features of the curve in figure 7 could be accounted for by the curve in figure 6 if the unstable regions of the latter were actually rather flattopped maxima instead of the saw-tooth shape dotted in the figure. If this explanation is correct, then the roughness of the HTAB monolayer in this case has, fortuitously, given us an extra clue about the full shape of the oscillatory force curve.

The peak-peak amplitude decays exponentially with distance, as shown in the inset to figure 7 . The decay length is $2.7 \mathrm{~nm}$, again close to the layer spacing.

3.3.2 Smectic liquid crystal. - At this point it is pertinent to mention some results obtained with 4'-n-octyl-4-cyanobiphenyl (8CB), which is a smectic-A at room temperature. An experiment was done in which $8 \mathrm{CB}$ was oriented homeotropically between the mica surfaces, by coating them with HTAB monolayers. The results are not shown here, but we will describe their salient features.

The force-distance curve is again oscillatory, but this time the oscillations do not decay to zero. The amplitude of the oscillations is large near contact, and decreases over the first four or five layers until it reaches a finite value which is apparently maintained indefinitely. The oscillations showed no signs of diminishing out to $2 \mu \mathrm{m}$ and beyond - a thickness of more than 600 smectic layers. At large separations individual oscillations become " softer " in the sense described in the previous section. In fact, a certain elasticity of the layers could be detected. Starting with a certain number of layers, the surfaces could be brought together for some distance - typically $1-2 \%$ of the total - before any layers were squeezed out. From then on they would move in a series of jumps, each jump corresponding to one layer. Similarly, on separating, the layers could be « stretched " a little before new layers were sucked in. The periodicity of the oscillations (averaged over many layers) is $3.10 \mathrm{~nm}$ on approaching (compressed layers) and $3.19 \mathrm{~nm}$ on separating (extended layers).

This behaviour for $8 \mathrm{CB}$ is entirely consistent with our interpretation of the 5CB results shown in figures 6 and 7 . We attributed the form of those force curves to smectic ordering of the 5CB molecules near the smooth mica surfaces; this ordering is maintained for only a few layers near each surface, and so the force oscillations die away after five or six layers. In $8 \mathrm{CB}$, on the other hand, the smectic ordering is long-range and we find oscillations in the force continuing indefinitely.

\subsubsection{Planar orientation. - The oscillatory force} curves described above are readily understood in terms of smectic ordering of the molecules in the homeotropic orientation. However, the phenomenon turns out to be more general : we have measured qualitatively similar curves with $5 \mathrm{CB}$ in the planar orientation.

Typical force curves are shown in figures 8 and 9. In figure 8 the oscillations are strong, and measurable over at least six periods, whereas in figure 9 they are weaker and only three were measurable. This is another illustration of how the forces can vary from 


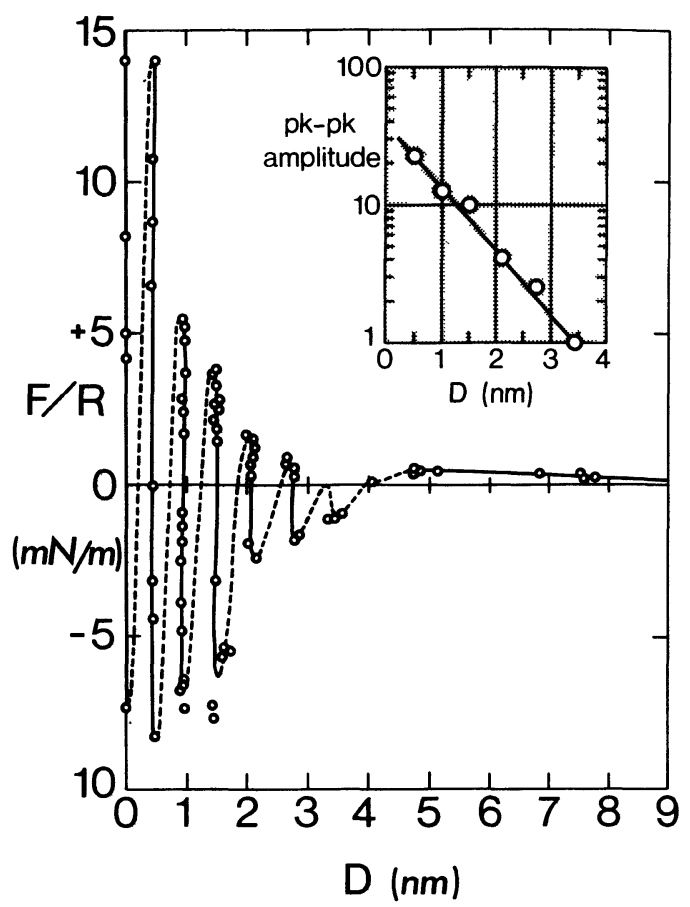

Fig. 8. - Strong short-range force for 5CB in the planar orientation. The exponential decay length of the peak-to-peak amplitude is $1.0 \mathrm{~nm}$ (inset). Note the smooth monotonic decay of the much weaker medium-range force at larger separations.

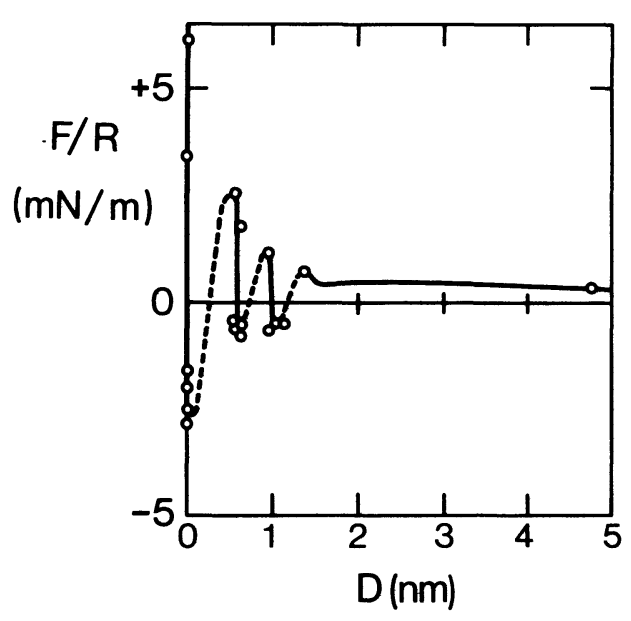

Fig. 9. - Weak short-range force for $5 \mathrm{CB}$ in the planar orientation.

experiment to experiment, presumably depending on the precise conditions at the mica-liquid crystal interface. In spite of this variability, oscillations are always observed.

By analogy with the homeotropic case, we ascribe these oscillations to a tendency of the molecules to be ordered in layers near the mica surface. However, this time they are not smectic layers, for the molecules lie with their long axes in the layers. While the idea of smectic layering is familiar to anyone with a knowledge of liquid crystals, perhaps the idea of layering in the planar orientation is more difficult to accept. However, it is no different in principle. Given a molecularly smooth solid surface, the molecules of a liquid next to it must form a layer, the molecules next to them will tend to form another layer, and so on. In fact this behaviour does not depend on our sample being a liquid crystal; we will see in the next section that the same phenomenon is observed in an isotropic liquid of spherical molecules.

The periodicity of the oscillations in the planar alignment is about $0.5 \mathrm{~nm}$, which corresponds to the average diameter of the 5CB molecule [21]. Close examination of figure 8 reveals a feature which was also found in other experiments : the first two or three oscillations have a periodicity of $0.5 \mathrm{~nm}$, but beyond that the periodicity stretches to about $0.6 \mathrm{~nm}$ - indicative, perhaps, of increased rotational motion of the molecules more than one or two layers from the surface.

In both figures 8 and 9 the short-range oscillatory force curve tails off at larger distances into the mediumrange monotonic force (Fig. 2). The inset in figure 8 shows the peak-to-peak amplitude of the oscillations plotted on a logarithmic scale against distance. Once again there appears to be an exponential decay, this time with a decay length of about $1.0 \mathrm{~nm}$ - about twice the layer spacing.

According to our intepretation, these oscillations result from a short-range positional ordering of the molecules near the surfaces, and should not depend on the long-range orientational order which characterizes the liquid crystal state. A test of this was provided by an experiment in which $5 \mathrm{CB}$, in the planar orientation, was heated to above the nematic-isotropic transition temperature. (In such thin films, the nematicisotropic transition becomes continuous, and the transition temperature is elevated slightly $[3,4]$.) In going from $22^{\circ} \mathrm{C}$ to $40^{\circ} \mathrm{C}$ we found that the force remained oscillatory, although the amplitude of the oscillations was reduced. Thus in contrast to the monotonic medium-range force which disappears above the transition, the positional ordering which gives rise to the oscillatory short-range force does persist into the isotropic phase, in accordance with the observations of Leadbetter et al. [21].

3.3.4 Experiments with isotropic liquids. Although oscillatory forces were seen in 5CB above the nematic-isotropic transition temperature, this phase is not necessarily isotropic in such thin films, because the molecular orientation induced by the surfaces persists for some distance into the liquid [3, 4].

We have performed similar experiments on two isotropic liquids composed of more or less spherical molecules, and once again found oscillations in the force-distance curve with periodicity equal to the molecular size. Between five and ten oscillations can be detected. The first results, for octamethylcyclotetrasiloxane, have been the subject of a recent brief report [23]; an article in preparation [24] will give full details of these and similar results for cyclohexane. 
4. Summary. - The forces we have measured between solid surfaces separated by a film of liquid crystal have contributions from three distinct effects, each of which can be considered as a type of structural force.

Two of these are measurable over a similar range (up to $80 \mathrm{~nm}$ ) : the familiar elastic force, and a force which we ascribe to the effects of a modified order parameter near the surfaces. The elastic force is dominated by the twist mode, and is only measurable in a planar sample twisted by a significant amount $\left(z 10^{\circ}\right)$. Contributions from splay and bend modes are negligibly small in the geometry of this experiment. This force results from the long-range orientational order in a nematic liquid crystal, and in principle it extends over a long range, falling off as $1 / D$. In practice its magnitude is small, and for the maximum twist angle used here $\left(33^{\circ}\right)$ it was only measurable below $100 \mathrm{~nm}$. Clearly, this force would only be found in a liquid crystal.

The force arising from the effects of the surfaces modifying the order parameter in the liquid crystal near them is of similar magnitude to the elastic force in a twisted sample in the range $10-50 \mathrm{~nm}$; it is found in both the planar and homeotropic orientations. Its range should be a measure of a type of correlation length : the distance over which a local variation in the magnitude of the order parameter in the nematic phase persists before it attains its bulk value. This correlation length is not necessarily the same as that associated with a local variation in the direction of alignment, which is the one usually considered in analyses of light scattering in nematics, although it may be comparable in size. The decay length of the curves in figure 2 is about $15 \mathrm{~nm}$.

The concept of a force due to a modified order parameter near surfaces is perhaps the best way to consider structural forces in general. In other systems a different order parameter (or a number of order parameters) could be defined to describe how the local state of the fluid is perturbed by a nearby surface ; and when two surfaces come close enough together for the perturbed regions to overlap, the free energy of the system may become a function of the separation between the surfaces, thus giving rise to a structural force. Here we believe we have demonstrated the existence of such a force, where the relevant order parameter is just the conventional orientational order parameter of a nematic liquid crystal. A theoretical account of this force requires a detailed consideration of all of the interactions involved in the system, and has not been attempted here.

A third type of force becomes dominant at very small distances. It arises from the positional ordering of liquid crystal molecules near surfaces, which leads to a force curve having oscillations whose period equals the molecular dimensions. The oscillations decay away within a few layers, the decay length giving a measure of the correlation length of positional ordering [21]. In the homeotropic orientation this behaviour is explained by a tendency of the 5CB molecules to form smectic layers near the smooth mica surfaces - an idea which is not new to people in the liquid crystal field $[5,6]$. However, we find very similar behaviour in the planar alignment, and indeed in an isotropic liquid of spherical molecules [23, 24], so the concept of layering does not depend on having a smectic phase. Any collection of identical objects could form a uniform layer adjacent to a smooth surface so long as they are all oriented in the same direction - indeed, if they are attracted to the surface, they would be expected to do so. The only property of the liquid crystal molecules which is crucial here is their habit of aligning parallel to each other. Oscillatory force-distance curves are a more general phenomenon arising from the molecular nature of liquids [1619].

As a final remark, we comment on the variability of all of the force curves, typified for example by the different curves in figure 2, or by comparing figures 6 and 7 and figures 8 and 9 . In this series of experiments the force curves were sometimes rather irreproducible, varying with all sorts of conditions such as the type of mica used, the exact position and orientation of the mica, humidity, time, and whether the two micas had previously been forced into molecular contact. But this is not surprising, since the structural forces we have been discussing depend on how much the ordering of the liquid crystal molecules is modified by the surfaces, which in turn depends on their exact physicochemical condition. The simplest example of this is that the twist elastic force depends on the relative orientation of the two mica sheets (Fig. 3). The other forces, resulting from orientational and positional ordering of molecules next to the mica, must depend on whatever intermolecular (anchoring) forces cause that ordering. These could be expected to vary between different HTAB monolayers, or from mica to mica, or along the surface of a mica sheet, since there are known to be inhomogeneities in its composition [7]. In addition, impurities (of which water is one) can diffuse gradually to the hydrophilic mica surfaces and remain there; and their state, or indeed the state of the surface itself, may be modified by pressure.

Such variability, not to mention the complexity of the surfaces, makes it a daunting task to attempt a detailed theoretical analysis of the results we have presented. Our aim has simply been to demonstrate that there are structural forces of different types across a film of nematic liquid crystal, and to illustrate their general features.

Acknowledgments. - The authors thank D. Y. C. Chan and S. Marčelja for helpful discussions, and the referees for their constructive comments. 


\section{References}

[1] Ninham, B. W., J. Phys. Chem. 84 (1980) 1423.

[2] De Gennes, P. G., The Physics of Liquid Crystals (Clarendon, Oxford) 1974.

[3] Sheng, P., Phys. Rev. Lett. 37 (1976) 1059.

[4] SCHRÖDER, H., J. Chem. Phys. 67 (1977) 16.

[5] Manev, E., Proust, J. E. and Ter-Minassian-Saraga, L., Colloid Polym. Sci. 255 (1977) 1133.

[6] Rosenblatt, C. and Amer, N. B., Appl. Phys. Lett. 36 (1980) 432.

[7] IsRaelachvili, J. N. and Adams, G. E., J. Chem. Soc. Faraday I 74 (1978) 975

[8] Israelachvili, J. N., J. Colloid Interface Sci. 44 (1973) 259.

[9] Shafrin, E. G. and Zisman, W. A., J. Colloid Sci. 4 (1949) 571.

[10] DerJaguin, B. V., Kolloid Z. 69 (1934) 155.

[11] Gillespie, T. and Rose, G. D., J. Colloid Interface Sci. 26 (1968) 246.

[12] Karat, P. P. and Madhusudana, N. V., Mol. Cryst. Liq. Cryst. 40 (1977) 239.

[13] Kléman, M. and Williams, C., Philos. Mag. 28 (1973) 725.
[14] Meyer, R. B., Solid State Commun. 12 (1973) 585.

[15] Horn, R. G., J. Physique 39 (1978) 105, 342.

[16] Mitchell, D. J., Ninham, B. W. and Pailthorpe, B. A., J. Chem. Soc. Faraday II 74 (1978) 1098, 1116.

[17] Snook, I. K. and van Megen, W., J. Chem. Phys. 72 (1980) 2907.

[18] Lane, J. E. and Spurling, T. H., Chem. Phys. Lett. 67 (1979) 107.

[19] Grimson, M. J., Rickayzen, G. and Richmond, P., Mol. Phys. 39 (1980) 61.

[20] Marčelua, S. and Radić, N., Chem. Phys. Lett. 42 (1976) 129.

[21] Leadbetter, A. J., Richardson, R. M. and Colling, C. N., J. Physique Colloq. 36 (1975) C1-37.

[22] De Gennes, P. G., C. R. Hebd. Séan. Acad. Sci. Paris B 275 (1972) 939 .

[23] Horn, R. G. and Israelachvili, J. N., Chem. Phys. Lett. 71 (1980) 192

[24] HoRN, R. G. and IsRaElaChVILI, J. N., to be published. 